\title{
Removal of haloacetonitrile by adsorption on thiol-functionalized mesoporous composites based on natural rubber and hexagonal mesoporous silica
}

\author{
Yaowalak Krueyai $^{1,2}$, Patiparn Punyapalakul ${ }^{2,3}$, Aunnop Wongrueng ${ }^{2,4^{\dagger}}$ \\ ${ }^{1}$ International Postgraduate Program in Hazardous Substance and Environmental Management, Graduate school, Chulalongkorn University, Bangkok \\ 10330, Thailand \\ ${ }^{2}$ Center of Excellence on Hazardous Substance Management, Chulalongkorn University, Bangkok 10330, Thailand \\ ${ }^{3}$ Department of Environmental Engineering, Faculty of Engineering, Chulalongkorn University, Bangkok 10330, Thailand \\ ${ }^{4}$ Department of Environmental Engineering, Faculty of Engineering, Chiang Mai University, Chiang Mai 50200, Thailand
}

\begin{abstract}
Haloacetonitriles (HANs) are nitrogenous disinfection by-products (DBPs) that have been reported to have a higher toxicity than the other groups of DBPs. The adsorption process is mostly used to remove HANs in aqueous solutions. Functionalized composite materials tend to be effective adsorbents due to their hydrophobicity and specific adsorptive mechanism. In this study, the removal of dichloroacetonitrile (DCAN) from tap water by adsorption on thiol-functionalized mesoporous composites made from natural rubber (NR) and hexagonal mesoporous silica (HMS-SH) was investigated. Fourier-transform infrared spectroscopy (FTIR) results revealed that the thiol group of NR/HMS was covered with NR molecules. X-ray diffraction (XRD) analysis indicated an expansion of the hexagonal unit cell. Adsorption kinetic and isotherm models were used to determine the adsorption mechanisms and the experiments revealed that NR/HMS-SH had a higher DCAN adsorption capacity than powered activated carbon (PAC). NR/HMS-SH adsorption reached equilibrium after 12 hours and its adsorption kinetics fit well with a pseudo-second-order model. A linear model was found to fit well with the DCAN adsorption isotherm at a low concentration level.
\end{abstract}

Keywords: Adsorption, Composite material, Haloacetonitrile

\section{Introduction}

Haloacetonotriles (HANs) are nitrogenous species of disinfection by-products (DBPs), which can be formed by reactions between chlorine, chloramine or bromine disinfectants and natural organic matter (NOM). HANs are of concern because they cause problems in wastewater and tap water. The disinfection of drinking water reduces microbial risks but increases chemical exposure to man. Increasing of human health risks are due to the formation of disinfection by-products (DBPs) in the organic and inorganic precursors. Monochloroacetronitrile (MCAN), dichloroacetonitrile (DCAN), trichloroacetonitrile (TCAN), bromochloroacetonitrile (BCAN), and dibromoacetonitrile (DBAN) are common HANs.

Several techniques can remove DBPs from water such as adsorption [1], ozonation [2] and membrane filtration [3]. Among these three processes, however, the adsorption process has been most popular for water treatment because of its low cost and simple application. Although different materials have been used to adsorb DBPs, a suitable one for use with tap water has not been identified. Consequently, a study on the removal HANs from tap water using modified adsorbents can be deemed useful.

Among porous materials, mesoporous silicas provide a number of advantages as adsorbents because of their high surface area and narrow pore size, which improves their adsorption capacity and adsorption selectivity. Moreover, hexagonal mesoporous silica (HMS) is one of many kinds of adsorbents that have been used. In the past, a polymer/silica composite has been studied as a new material that has the advantages of silica and organic polymer. Natural rubber (NR) is an organic material that many have attempted to modify because of its thermal stability and mechanical properties. A NR/HMS composite has been synthesized and characterized as an attractive prospective adsorbent.

Consequently, the objectives of this study are to investigate the adsorption efficiency of a thiol-functionalized mesoporous com-
This is an Open Access article distributed under the terms of the Creative Commons Attribution Non-Commercial License (http://creativecommons. org/ licenses/by-nc/3.0/) which permits unrestricted non-commercial use, distribution, and reproduction in any medium, provided the original work is properly cited.
Received July 15, 2015 Accepted September 4, 2015

${ }^{\dagger}$ Corresponding author

Email: aunnop@eng.cmu.ac.th

Tel: +66-86-404-2730 Fax: +66-53-210-328

Copyright $@ 2015$ Korean Society of Environmental Engineers 
posite made from natural rubber and hexagonal mesoporous silica (NR/HMS-SH) in haloacetonitrile (HANs) removal. In this study, dichloroacetonitrile (DCAN) was selected as the representative HAN because it is a chloro-DBP that is highly toxic and has a considerable presence in water. The modification of hexagonal mesoporous silica (HMS) with the thiol group (R-SH) and natural rubber was employed to investigate the effect of surface functional groups on the DCAN adsorption. The adsorption experiments were carried out in batch experiments. The adsorption kinetics and the adsorption isotherm were studied to investigate the adsorption mechanisms. Moreover, the ionic strength and $\mathrm{pH}$ of synthesized water was controlled so that it could adequately represent tap water in this study.

\section{Materials and Methods}

\subsection{Preparation of NR/HMS-SH}

\subsubsection{Materials and reagents}

TEOS (AR grade, >99\%), MPTMS (AR grade, 95\%), and DDA (AR grade, 98\%) were purchased from Sigma-Aldrich (Germany), THF (AR grade, 99.5\%), $\mathrm{C}_{2} \mathrm{H}_{5} \mathrm{OH}$ (AR grade, 99.9\%), and $\mathrm{H}_{2} \mathrm{SO}_{4}$ (AR grade, 98\%) were purchased from QRëC (New Zealand). The NR (commercial grade) was supplied by the Thai Hua Chumporn Natural Rubber Co., Ltd. (Thailand).

\subsubsection{Synthesis of NR/HMS-SH}

The thiol-functionalized mesoporous composite was made of natural rubber and hexagonal mesoporous silicate (NR/HMS-SH) according to the condition of NR/HMS- $\mathrm{SO}_{3} \mathrm{H}$ synthesis [4]. Firstly, $1 \mathrm{~g}$ of a natural rubber (NR) sheet was mixed with 30 $\mathrm{mL}$ of tetrahydrofuran (THF) at room temperature overnight. The NR sheet was completely dissolved in the THF to obtain a homogeneous solution. Secondly, $7.51 \mathrm{~g}$ of dodecylamine (DDA) was added in the solution and stirred. After $0.5 \mathrm{~h}, 21$ $\mathrm{g}$ of tetraethoxysilane (TEOS) was added and stirred for another $0.5 \mathrm{~h}$. Next, $106 \mathrm{~g}$ of $\mathrm{H}_{2} \mathrm{O}$ was added and stirred at $40^{\circ} \mathrm{C}$ for $0.5 \mathrm{~h}$ and then $4.96 \mathrm{~g}$ of 3-mercaptopropyltrimethoxysilane (MPTMS) was added into the mixture by stirring and then it was left to stand at $40^{\circ} \mathrm{C}$ for $1 \mathrm{~h}$. The gel was aged at $40^{\circ} \mathrm{C}$ for 3 days, after which it was precipitated in $100 \mathrm{~mL}$ of ethanol. The solid product was vacuum dried at $60^{\circ} \mathrm{C}$ for $2 \mathrm{~h}$. Finally, the template in the composite was removed with $0.05 \mathrm{M}$ of $\mathrm{H}_{2} \mathrm{SO}_{4} / \mathrm{EtOH}$ that from the concentrated sulfuric acid was dissolved in the $99.9 \%$ ethanol at $70^{\circ} \mathrm{C}$ for $8 \mathrm{~h}$ and filtered; it was then vacuum dried with ethanol. The $\mathrm{pH}$ was checked at around 7 and dried at $80^{\circ} \mathrm{C}$ overnight.

\subsection{Characterization of NR/HMS-SH}

Structural information on the NR/HMS-SH composite was obtained using X-ray powder diffraction (XRD) analysis on X-ray power of $40 \mathrm{kV}$ and $40 \mathrm{~mA}$. The repeating distance between the pore centers of the hexagonal structure was calculated from the XRD data [5]. A Fourier-transform infrared (FT-IR) spectrometer equipped with a mercury cadmium telluride detector was applied for the identification of NR and the functional groups in the composite. A self-supporting disk (20 mm, 10-20 mg) was placed in the quartz cell attached to a conventional closed circulation system. All the IR spectra were recorded under evacuation at $25^{\circ} \mathrm{C}$. A total of 64 scans over $400-4000 / \mathrm{cm}$ at a resolution of $4 / \mathrm{cm}$ was averaged for each spectrum.

The particle size of the adsorbent was taken by using a scanning electron microscope (SEM). The sample was fixed on an aluminum stub before it was observed by the SEM. The samples on the copper grids were observed without any metal coating.

\subsection{Adsorption Study}

Stock solutions of HANs were prepared in deionized water, and a phosphate buffer was used to adjust the $\mathrm{pH}$ of the solution to 7 and ionic strength (IS) to $10 \mathrm{mM}$. The kinetic study was prepared by varying the contact time from 0 to $36 \mathrm{~h}$. DCAN was selected as the model HAN. The initial DCAN concentration was $1 \mathrm{mg} / \mathrm{L}$ at $\mathrm{pH}$ 7. After that, $0.025 \mathrm{~g}$ of the adsorbent and $50 \mathrm{~mL}$ of the DCAN solution were mixed in a $125 \mathrm{~mL}$ Erlenmayer flask and covered with aluminium foil. The mixture was stirred at $250 \mathrm{C}$ and the supernatant solution was filtrated through a nylon syringe filter (pore size $0.45 \mathrm{~mm}$ ). The concentration of DCAN was analyzed by gas chromatography with an electron capture detector (GC/ECD) according to EPA Method 551.1 [6]. The concentrations in the isotherm studies were varied from 0.05 to $2 \mathrm{mg} / \mathrm{L}$ in single solutions at $\mathrm{pH} 7$, while the ionic strength, adjusted by a phosphate buffer, remain fixed at $10 \mathrm{mM}$. The contact time was obtained from the kinetic study. After reaching equilibrium, the mixture was separated using a nylon filter and the solution was analyzed by gas chromatography with an electron capture detector (GC/ECD).

\section{Results and Discussion}

\subsection{Physiochemical Characteristics of the Synthesized Adsorbents}

\subsubsection{XRD analysis}

Fig. 1 shows the XRD pattern of the NR/HMS-SH composite after the extraction of the template molecules. This material exhibited one diffraction peak at $2 \theta$ in the $2.2^{\circ}$, corresponding to the characteristics of the hexagonal porous structure.

\subsubsection{FTIR spectroscopy}

FTIR analysis was used to confirm the presence of NR and the $\mathrm{SH}$ functional group in the HMS structure of the NR/HMS-SH composites (Fig. 2). The stretching vibration of the silica framework (Si-O-Si) appeared between 1000 and 1300/cm. The broad band at around $3500 / \mathrm{cm}$ can be assigned to the free silanol group. The band related to the thiol group was observed at around $2550-2620 / \mathrm{cm}(\mathrm{S}-\mathrm{H})$, but it was a very weak band.

\subsubsection{Electron microscopy}

The particle size of a NR/HMS-SH composite is shown in Fig. 3. The presence of NR/HMS-SH enhanced the agglomeration of HMS particles. It was observed that NR was homogenously dispersed throughout the particles of the NR/HMS composite. 


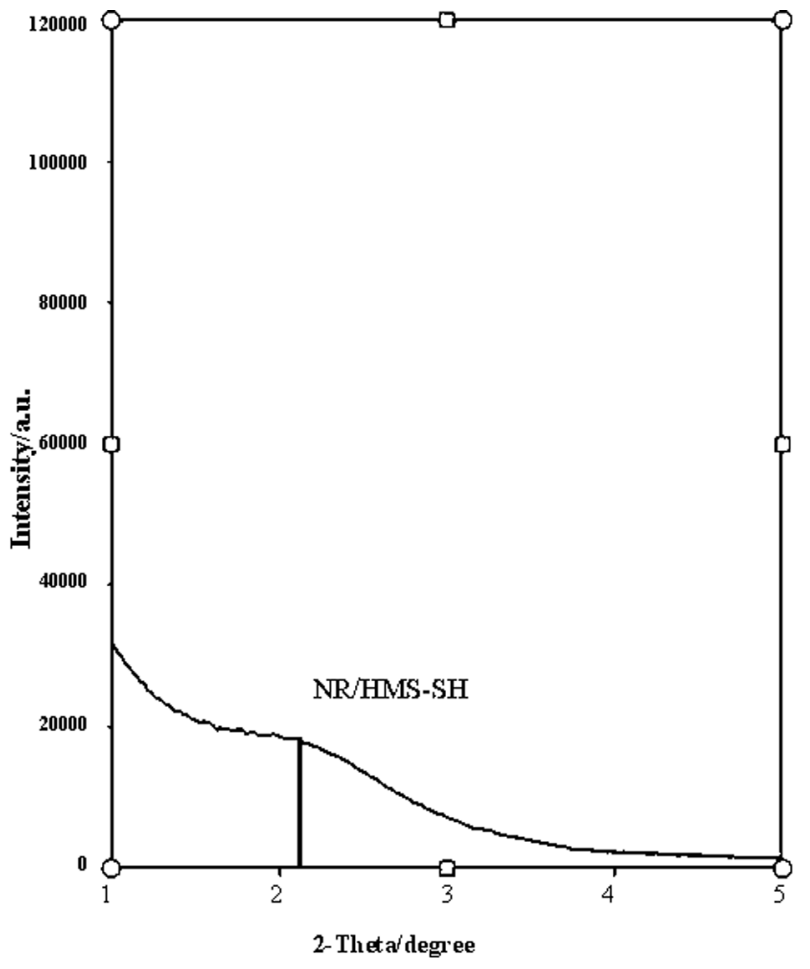

Fig 1. XRD pattern of the NR/HMS-SH that was extracted for removing the DDA template.

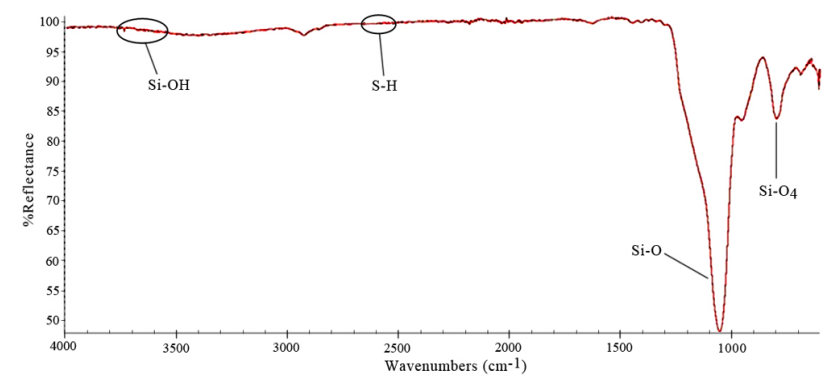

Fig. 2. FTIR spectra of NR/HMS-SH.

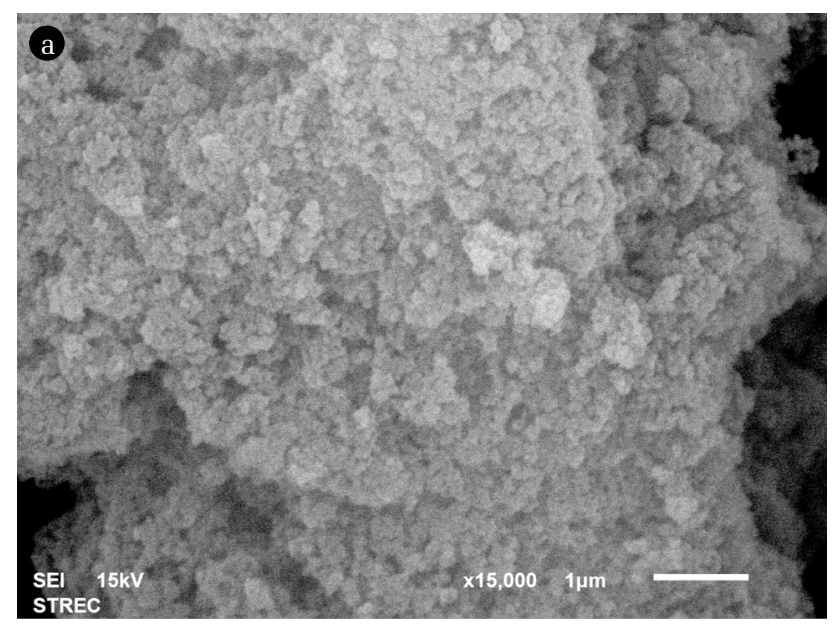

\subsection{Adsorption Kinetics}

The kinetic curve for DCAN adsorption on the porous adsorbent is shown in Fig. 4. A large amount of DCAN was adsorbed over PAC and NR/HMS-SH, but with PAC equilibrium was reached after a short contact time (2 h). For NR/HMS-SH, the amount of adsorbed DCAN reached equilibrium at $12 \mathrm{~h}$.

Kinetic modeling and pseudo-first-order and pseudo-second-order models were employed to investigate the adsorption mechanisms. Pseudo-first-order and pseudo-second-order equations can be defined as shown by Eqs. (1) and (2).

$$
\begin{gathered}
q_{t}=q_{e}\left(1-\exp ^{\left(-k_{1} t\right)}\right) \\
\frac{t}{q_{t}}=\frac{1}{k_{2} q_{e}^{2}}+\frac{t}{q_{e}}
\end{gathered}
$$

where $q_{e}$ is the amount of adsorbed contaminant at equilibrium (mg/g), $q_{t}$ is the amount of adsorbed contaminant at time $t$ (mg/g), $k_{1}$ is the rate constant of pseudo-first-order adsorption $\left(\mathrm{min}^{-1}\right)$, and $k_{2}$ is the rate constant of pseudo-second-order adsorption (g/mg.min).

Based on the pseudo-second-order model, the initial adsorption rate can be determined using Eq. (3).

$$
h=k_{2} q_{e}^{2}
$$

In order to quantitatively compare the applicability of different kinetic models, a normalized standard deviation $\triangle q$ (\%) was used as shown in Eq. (4).

$$
\triangle q(\%)=100 \times \sqrt{\frac{\left.\sum\left[q_{\mathrm{exp}}-q_{\text {cal }}\right) / q_{\mathrm{exp}}\right]^{2}}{N-1}}
$$

where $N$ is the number of data points and $q_{\exp }$ and $q_{c a l}(\mathrm{mg} / \mathrm{g})$ are the experimental and the calculated adsorption capacities. The best fit models should have the lowest $\triangle q$ (\%) values. The kinetic parameters were calculated and the results are shown in Table 1.

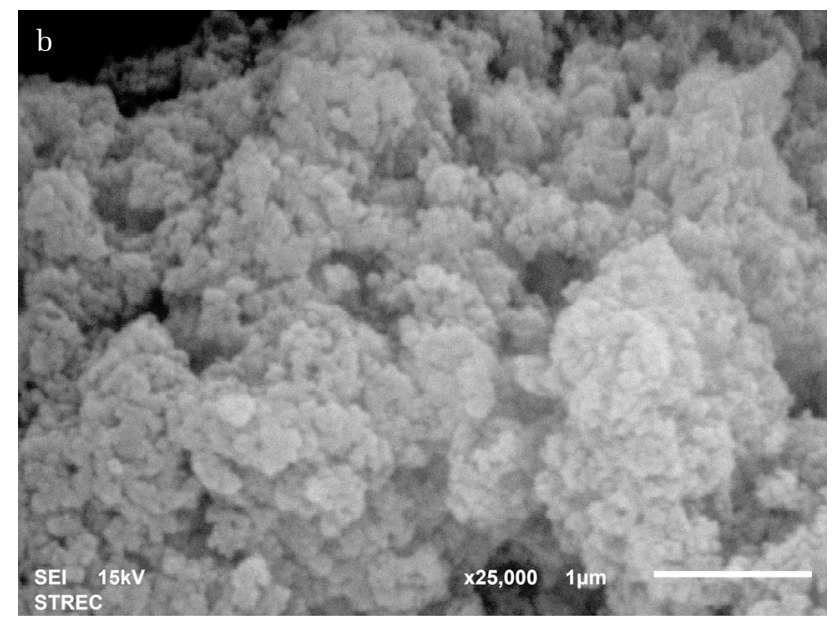

Fig. 3. SEM image of NR/HMS-SH at a magnification (a) $x 15,000$ and (b) $x 25,000$. 
Table 1. Kinetic Parameters of DCAN Adsorption on NR/HMS-SH using Pseudo-first-order and Pseudo-second-order Kinetic Models

\begin{tabular}{|c|c|c|c|c|c|c|c|c|}
\hline Adsorbent & $\begin{array}{c}\mathbf{q}_{\mathrm{e}}, \exp \\
(\mu \mathrm{g} / \mathrm{g})\end{array}$ & $\begin{array}{c}\text { h } \\
(\mu \mathrm{g} / \mathrm{g} \cdot \min )\end{array}$ & $\begin{array}{l}q_{\mathrm{e}}, \mathbf{c a l} \\
(\mu \mathrm{g} / \mathrm{g})\end{array}$ & $\begin{array}{c}\mathbf{k}_{\mathbf{1}} \\
(1 / \mathrm{min})\end{array}$ & $\begin{array}{c}\mathrm{k}_{2} \\
(\mathrm{~g} / \mathrm{mg} \cdot \min )\end{array}$ & $\mathbf{R}^{2}$ & $\triangle q(\mathbf{6})$ & Kinetic model \\
\hline \multirow{2}{*}{ NR/HMS-SH } & \multirow{2}{*}{274} & \multirow{2}{*}{6.13} & 260 & 0.0042 & - & 0.8173 & 65.23 & pseudo-first-order \\
\hline & & & 268 & - & $7.51 \times 10-5$ & 0.9969 & 27.22 & pseudo-second-order \\
\hline
\end{tabular}

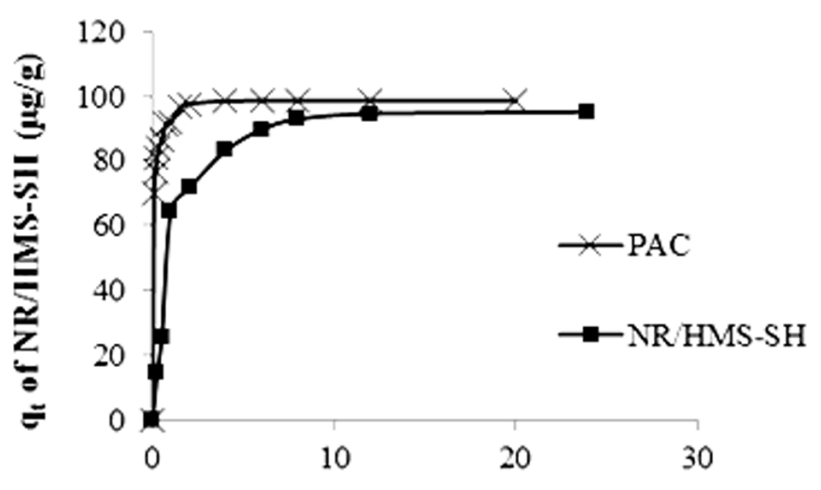

Time (hr)

Fig. 4. DCAN adsorption kinetics of PAC and NR/HMS-SH at initial concentration $100 \mu \mathrm{g} / \mathrm{L}, \mathrm{pH}$ 7, IS $=0.01 \mathrm{M}$.

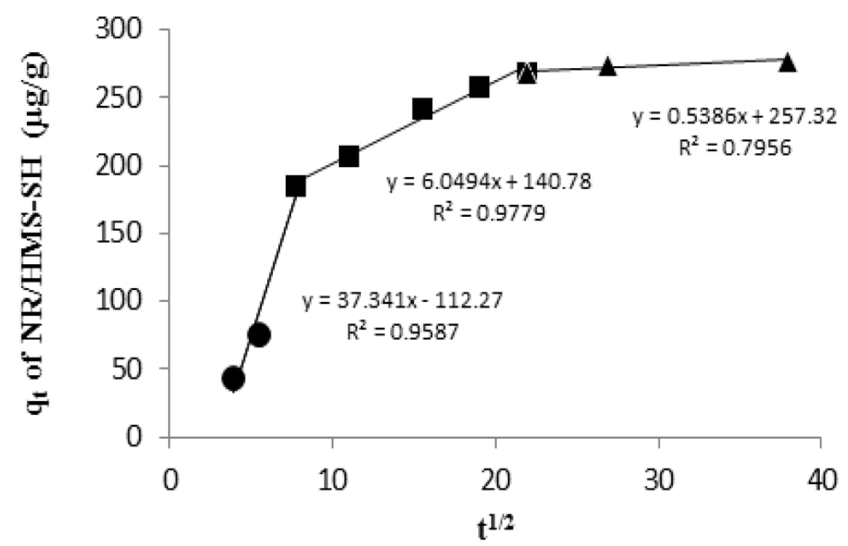

Fig. 5. Plot of the intraparticle diffusion model for the adsorption of DCAN on NR/HMS-SH.

The data fitting curve for NR/HMS-SH is shown in Fig. 5. It displays three regimes; the first regime presents the external mass transfer in the boundary layer and the second presents the diffusion through the pores of the adsorbent. Moreover, the last step presents the DCAN adsorption on the internal site of the adsorbent. It occurred very quickly, so the rate limiting step of the adsorption must have happened during the first or the second step. Both steps together could thus be considered the controlling step.

\subsection{Adsorption Isotherms}

NR/HMS-SH is a functionalized silica-based material that had a comparable adsorption capacity to PAC. Moreover, the results indicate that increasing the specific surface area of the adsorbent cannot be used as an explanation for DCAN's enhanced adsorption capacities when compared with the results of a previous study, which gave a similar specific surface area for HMS that functionalized with the thiol group without adding the natural rubber (SBET NR/HMS-SH of $880.4 \mathrm{~m}^{2} / \mathrm{g}$ ). The results, however, can be used to explain the density of the functional group because NR/HMS-SH provided less density of sulfur than HMS in the previous study [7]. It can be argued that the natural rubber in the adsorbent may have affected the interaction that occurred between the functional group and HMS.

In order to model the adsorption mechanism, linear, Langmuir and Freundlich isotherm models were used to test the experimental adsorption process. The linear, Langmuir and Freundlich equations can be defined as shown in Eqs. (5), (6) and (7).

$$
\begin{gathered}
q_{e}=K_{p} C_{e} \\
\frac{1}{q_{e}}=\frac{1}{K_{L} q_{m} C_{e}}+\frac{1}{q_{m}} \\
q_{e}=K_{F} C_{e}^{1 / n}
\end{gathered}
$$

where $q_{e}$ is the amount of adsorbate adsorbed at equilibrium (mg/g), $K_{p}$ is the linear constant (L/mg), $q_{m}$ is the maximum adsorption capacity (mg/g), and $K_{L}$ is the Langmuir constant (L/mg). $K_{F}$ and $\mathrm{n}$ are constants, $C_{e}$ is the equilibrium concentration $(\mathrm{mg} / \mathrm{L})$, and $C_{e}$ is the concentration of the adsorbate at equilibrium (mg/L).

The isotherm parameters of DCAN adsorption on NR/HMS-SH are listed in Table 2. Meanwhile, the predicted and experimental data for the equilibrium adsorption of DCAN on NR/HMS-SH are shown in Fig. 6.

Table 2. Isotherm Parameters of the DCAN Adsorption on the NR/HMS-SH Adsorbent

\begin{tabular}{ccc}
\hline \multicolumn{1}{c}{ Isotherms } & Langmuir & NR/HMS-SH \\
\hline$q_{m} \quad(\mathrm{mg} / \mathrm{g})$ & & - \\
$K_{L}$ & $(\mathrm{~L} / \mathrm{mg})$ & - \\
$R^{2}$ & & 0.6729 \\
$q(\%)$ & & 235.39 \\
\hline \multicolumn{1}{c}{ Freundlich } \\
$K_{F}(\mathrm{mg} / \mathrm{g})$ & & 1.6391 \\
$R^{2}$ & & 872.3685 \\
$q(\%)$ & & 0.7861 \\
\hline \multicolumn{2}{c}{} & 35.70 \\
\hline$K_{p}$ & Linear & 102.48 \\
$R^{2}$ & & 0.8961 \\
\hline
\end{tabular}




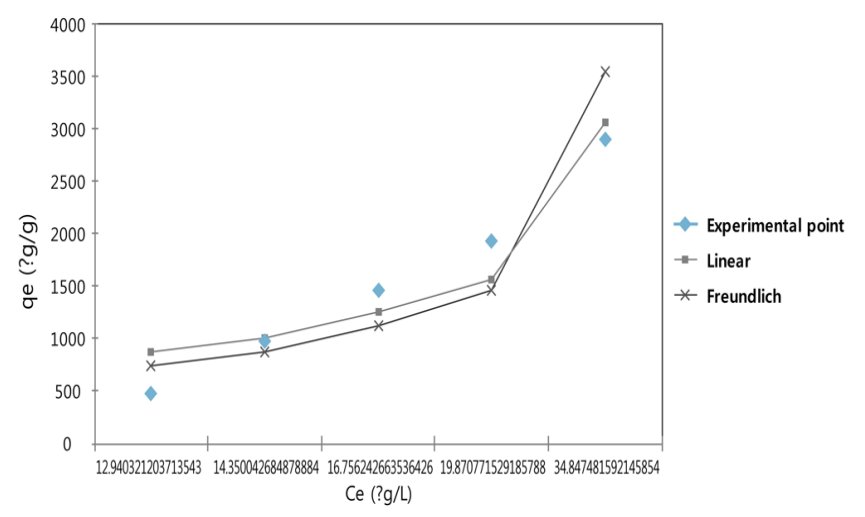

Fig. 6. Comparison of the predicted and experimental data for the equilibrium adsorption of DCAN on the NR/HMS-SH adsorbent.

\section{Conclusions}

A NR/HMS-SH composite with a high structure order and mesoporosity was successfully prepared. The formation of the NR/HMS-SH composite induced the coalescence of HMS nanoparticles, resulting in enhanced textural porosity and hydrophobicity. DCAN adsorption on the NR/HMS-SH adsorbent followed a pseudo-second order rate kinetic model. A linear model fit well with the DCAN adsorption isotherm at a low concentration level.

\section{Acknowledgements}

The authors gratefully acknowledge the financial support for this study from the International Postgraduate Program in Hazardous Substance and Environmental Management, under the Graduate School of Chulalongkorn University. This research was also supported by the National Research University Project, under the Office of the Higher Education Commission (WCU-014-FW-57). This work was carried out as part of a research cluster on the "Fate and Removal of Emerging Micropollutants in the Environment" under a grant by the Center of Excellence for Environmental and Hazardous Waste Management (EHWM) and Special Task Force for Activating Research (STAR), of Chulalongkorn
University and conducted as part of a research program on the "Control of Residual Hormones and Antimicrobial Agents from Aquacultural and the Feedstock Industry" under a grant from the Center of Excellence on Hazardous Substance Management (HSM). Technical support was provided by the Department of Environmental Engineering, under the Faculty of Engineering, of Chulalongkorn University and the Department of Chemical Technology, under the Faculty of Science, of Chulalongkorn University. The authors finally wish to thank Asst. Prof. Dr. Chawalit Ngamcharussrivichai and Mr. Sakdinun Nuntang for their insightful suggestions.

\section{References}

1. Uyak V, Yavuz S, Toroz I, Ozaydin S, Genceli EA. Disinfection by-products precursors removal by enhanced coagulation and PAC adsorption. Desalination 2007;216:334-344.

2. Vaiopoulou E, Misiti TM, Pavlostathis, SG. Removal and toxicity reduction of naphthenic acids by ozonation and combined ozonation-aerobic biodegradation. Bioresour. Technol. 2015; 179:339-347.

3. Uyak V, Koyuncu I, Oktem I, Cakmakci M, Toroz I. Removal of trihalomethanes from drinking water by nanofiltration membranes. J. Hazard. Mater. 2008;152:789-794.

4. Nuntang S, Poompradub S, Butnark S, Yokoi T, Tatsumi T, Ngamcharussrivichai C. Organosulfonic acid-functionalized mesoporous composites based on natural rubber and hexagonal mesoporous silica. Mater. Chem. Phys. 2014;147:583-593.

5. Tanev PT, Pinnavaia TJ. Mesoporous Silica Molecular Sieves Prepared by Ionic and neutral Surfactant Templating: A Comparison of Physical Properties. Chem. Mater. 1996;8: 2068-2079.

6. Hodgeson JW, Cohen AL. Determination of Chlorination Disinfection Byproducts, Chlorinated Solvents and Halogenated Pesticides/herbicides in Drinking Water by Liquid-Liquid Extraction and Gas Chromatography with Electron-capture Detection. Ohio: National Exposure Research Laboratory; 1990.

7. Prarat P, Ngamcharussrivichai C, Khaodhiar S, Punyapalakul P. Adsorption characteristics of haloacetonitriles on functionalized silica-based porous materials in aqueous solution. J. Hazard. Mater. 2011;192:1210-1218. 